\title{
Early smoking-onset age and risk of cardiovascular disease and mortality
}

Manel Fa-Binefa ${ }^{1,2}$; MS, Albert Clará ${ }^{1,3,4,5}$, PhD-MD; Silvia Pérez-Fernández ${ }^{4,5}$; Maria $\mathrm{Grau}^{4,5}, \mathrm{PhD}-\mathrm{MD}$; Irene R Dégano ${ }^{4,5,6}, \mathrm{PhD} ;$ Ruth Marti-Lluch ${ }^{7,8,9}, \mathrm{PhD}$; Rafel

$\operatorname{Ramos}^{7,8,9,10}$, PhD-MD; Jaume Marrugat J ${ }^{4,5}$, PhD-MD; Roberto Elosua ${ }^{4,5,6}$, PhD-MD

1.-School of Medicine, Universitat Autònoma de Barcelona, Barcelona, Spain

2.-School of Health and Life Sciences, Universitat Pompeu Fabra, Barcelona, Spain

3.-Department of Angiology and Vascular Surgery, Hospital del Mar, Barcelona, Spain

4.-Cardiovascular Epidemiology and Genetics Group, REGICOR, IMIM (Instituto Hospital del Mar de Investigaciones Médicas), Barcelona, Spain

5.-Center for the Biomedical Research Network in Cardiovascular Diseases (CIBERCV),

Barcelona, Spain

6.-Department of Medicine, Faculty of Medicine, Universitat de Vic-Central de Catalunya, Vic, Spain

7.-Unitat de Suport a la Recerca de Girona, Institut Universitari d'Investigació en Atenció

Primària Jordi Gol (IDIAP Jordi Gol), Girona, Spain

8.-ISV Research Group, Research Unit in Primary Care, Primary Care Services, Girona.

Institut Català de la Salut (ICS), Catalonia, Spain

9.-Biomedical Research Institute, Girona (IdIBGi). ICS, Spain

10.-Department of Medical Sciences, School of Medicine, University of Girona, Spain

\section{Corresponding author:}

Roberto Elosua

Cardiovascular Epidemiology and Genetics

IMIM, Dr Aiguader 88, 08003 Barcelona, Spain

Email: relosua@imim.es

Word count: Abstract 250 words; Text 3092 words; 2 Tables; 1 Figure Appendix: 4 Tables and 2 Figures 


\section{ABSTRACT}

Early smoking onset age (SOA) is a public health concern with scant empirical evidence of its role in health outcomes. The study had two aims: i) to assess whether an early SOA was associated with the risk of fatal and non-fatal CVD and all-cause and CVD mortality and ii) to explore the linear and non-linear association between SOA and the outcomes of interest. Data from 4,499 current or former smokers, recruited from 1995 to 2005 , aged 25 to 79 years, and with a median 7.02 years of follow-up, were obtained from the REGICOR population-based cohort. In the present analysis, performed in 2018, the independent variable was SOA and the dependent variables were CVD events, CVD mortality, and all-cause mortality. Penalized smoothing spline methods were used to assess the linear and non-linear association. During follow-up, 361 deaths and 210 CVD events were recorded. A significant non-linear component was identified in the association between SOA and CVD outcomes with a cut-off point at 12 years: In the group aged $\leq 12$ years, each year of delay in SOA was inversely associated with CVD risk $(\mathrm{HR}=0.71 ; 95 \% \mathrm{CI}=0.53-0.96)$ and $\mathrm{CVD}$ mortality $(\mathrm{HR}=0.58$; $95 \% \mathrm{CI}=0.37-0.90)$. No association was observed in the older SOA group. A linear association was observed between SOA and all-cause mortality, and each year of delay was associated with $4 \%$ lower risk of mortality $(\mathrm{HR}=0.96 ; 95 \% \mathrm{CI}=0.93-0.98)$. The associations were adjusted for lifelong exposure to tobacco and cardiovascular risk factors. These results reinforce the value of preventing tobacco use among teenagers and adolescents.

Keywords: Smoking, Youth, Cardiovascular diseases, Cardiovascular risk, Mortality, Smoking onset age 


\section{INTRODUCTION}

Consistent evidence supports the role of smoking as a risk factor for cardiovascular disease (CVD) (1-3). Smokers have about twice the risk of coronary heart disease (4) and stroke (5), compared to non-smokers. Smoking is also a risk factor for ischemic nephropathy (6), bowel ischemia (7) aortic dissection (8), cancer and all-cause mortality (9). Furthermore, smoking has been described as nearly a prerequisite for the development of peripheral arterial disease (10) and abdominal aortic aneurysms (11).

Several smoking indicators have been explored and analysed, with particular attention to smoking status (current, former and never smokers) and cumulative exposure to tobacco (1213). Early smoking-onset age (SOA) is another important indicator of exposure, as $68.1 \%$ of smokers in Europe start before 18 years of age and the mean age for onset of regular smoking is 16.6 years (14). An early SOA has been associated with psychiatric disorders (15), asthma (16), lung cancer and other malignancies (17-23) and all-cause mortality (12,20,23). Although some studies suggest a positive association between early SOA and CVD (20,24-26), potential limitations include a lack of adjustment for cumulative tobacco exposure (26) or vascular risk factors $(20,24,26)$, a retrospective design $(20,25)$ and under-representation by sex $(12,23,25)$. Moreover, the pattern of dose-response relationships between SOA and health outcomes has not been fully explored.

The study had two aims: i) to assess whether an early SOA was associated with the risk of fatal and non-fatal CVD and all-cause and CVD mortality and ii) to explore the linear and non-linear association between SOA and the clinical outcomes of interest in a Mediterranean population in southern Europe.

\section{MATERIAL AND METHODS}

\section{Study design and population}


The REGICOR (REgistre GIroni del COR, or Girona Heart Registry) study recruited a prospective population-based cohort in Girona province $(\sim 700,000$ inhabitants $)$ in northeastern Spain, with the objective of studying CVD and related risk factors over time. Recruitment details have been described elsewhere (27). Briefly, individuals living in 42 communities, including 41 villages and the city of Girona, were randomly selected from the census and invited to participate. Inclusion criteria required that participants were free of terminal disease, not institutionalized, and had lived in the referral area for at least six months/year (reflecting the stable seasonal presence of a large number of retirees).

Participants were recruited for three different surveys: 1,748 residents aged 25 to 74 years in $1995,3,058$ aged 25 to 74 years in 2000 , and 6,352 aged 35 to 79 years in 2005. Selected participants received a letter informing them of the overall aims of the study, the purpose of the specific survey, and the tests to be performed. The participation rates in these surveys were $72.4 \%, 70.0 \%$ and $73.8 \%$, respectively.

The present analysis included participants from all three surveys. In the case of participants in more than one survey, the longest available follow-up data were considered. We excluded those participants with personal history of CVD and those older than 79 years at the date of study inclusion, or reporting never having smoked. As very few smokers started before 9 years or after 30 years of age, these individuals were also excluded from analysis. From an initial sample of 11,158 individuals, 4,499 were finally included in the main analysis as shown in flow-chart (Figure 1). The study protocol was approved by the Parc de Salut Mar Research Ethics Committee (2008/3046/I; 2016/7075/I) and each participant signed an informed consent.

\section{Baseline data}


Participants were asked to fast for at least 10 hours before their appointment at the examination site, which included drawing a blood sample. A group of nurses trained in the study protocol administered a set of validated and standardized questionnaires $(28,29)$ and performed a physical examination.

Self-reported educational level (elementary school, secondary school or university degree) was considered as an indicator of socioeconomic position. Hypertension was defined if the individual was previously diagnosed by a physician or receiving treatment or presented with systolic blood pressure $(\mathrm{SBP}) \geq 140 \mathrm{mmHg}$ or diastolic blood pressure (DBP) values $\geq 90$ mmHg. Total cholesterol, high density lipoprotein (HDL) cholesterol, triglycerides and glucose concentrations were determined by enzymatic methods. When triglycerides were $<300 \mathrm{mg} / \mathrm{dL}$, low density lipoprotein (LDL) cholesterol was estimated by the Friedewald formula. Diabetes was defined if previously diagnosed or treated or when an individual presented with fasting glucose values $\geq 126 \mathrm{mg} / \mathrm{dL}$.

\section{Smoking data}

Smoking exposure was assessed by a standardized questionnaire $(28,29)$. Participants were classified as smokers (current or quit $<1$ year) or former smokers (quit $>1$ year). SOA was defined as the reported age at which the individual regularly smoked at least 1 cigarette/day. Lifetime pack-years was estimated as years of smoking multiplied by number of 20-cigarette packs consumed daily. Cigar smoking was included in the analysis according to tobacco equivalence to cigarettes (30). Pipe smoking was negligible within our population and was not considered.

Among current smokers (51.9\% of participants), years of smoking were quantified as the difference between age at the REGICOR survey visit and SOA. Among former smokers (48.1\% of participants), smoking exposure time was calculated as the difference between 
cessation age and SOA. As the type of information available about smoking cessation date varied between the three REGICOR surveys, the exact year former smokers quit smoking was available for $35.2 \%$ of participants. In those with missing data, we used multiple imputation methods implemented in the mice R package and obtained 20 data sets to impute the value of this variable. To obtain an estimator of the associations of interest we used the MIcombine function in $\mathrm{R}(31)$.

\section{Follow-up and outcomes}

The follow-up included physical re-exams and contact by telephone every two years. The re-exams included physical examination and all previously administered questionnaires. In those not attending the physical re-exams, a follow-up telephone contact was attempted to identify the appearance of events of interest, by means of a standard questionnaire. To ascertain any cardiovascular events or deaths, we also reviewed medical records, linked the data with a population-based myocardial infarction register, and cross-checked all these sources of information. To identify fatal events not otherwise reported, we linked our data with the regional mortality register (until 31 December 2010).

Three main outcomes were defined: i) CVD events, including non-fatal myocardial infarction or stroke and fatal CVD events (ICD9 codes: 390-459, 798 or ICD10 codes: I00I99, R96, R98-99), ii) CVD mortality, and iii) all-cause mortality. All the events were classified by an event committee according to standardized criteria (32). In case of multiple CVD events in the same participant, the first occurring event was considered in defining the composite CVD outcome.

\section{Statistical analysis}


The analysis was performed in 2018. Continuous variables were expressed as mean and standard deviation or median and interquartile range, and categorical data as frequencies and percentages. Categorical variables were compared with the Chi-square or Fisher exact test, as appropriate, and continuous variables with the t-test or ANOVA for normal distribution and the Mann-Whitney U-test or Kruskall-Wallis test for non-normal distributed variables.

To explore the hypothesis that an early SOA was independently associated with an increased risk of CVD event or mortality, we used penalized smoothing spline (pspline function, R Survival Package) which allows a maximum of 10 knot points, to assess the linear and non-linear components of the dose-response association (33). When the non-linear component was nonsignificant, SOA was considered as a continuous variable in Cox proportional hazard regression models. Otherwise, we used bootstrapping methods to define the best cut-point(s) at which a change in the linear dose-response association was observed. We performed 1000 iterations per outcome of interest and calculated the median of the observed cut-point, which was then defined as the best cut-point. The analysis was stratified according to best cut-point, and Cox regression modelling considered SOA as a continuous variable in each of the defined strata.

The assumption of the proportionality of risks according to the Schoenfeld residuals was checked in each Cox regression model. To control for potential confounding factors, all variables related to the outcomes of interest with a $p<0.05$ in bivariate analyses were considered in the multivariate model. Statistically non-significant variables were removed step-by-step from the model using a backward procedure when non-significant variations occurred in the regression coefficients of the SOA. Lifetime smoking exposure (pack-years), smoking status (current vs former smokers), years since quitting smoking, and recruitment survey were included in all models regardless of significance. The statistical analysis adopted a competing risk strategy using the Gray method, considering non-CVD death causes as the 
competing event for a CVD event or CVD-related mortality, and other death causes as the competing event for CVD-related mortality. As a sensitivity analysis, the results were stratified by current smoking status and by sex.

A p-value $<0.05$ was considered as statistically significant. All analyses were performed using the R statistical package (31).

\section{RESULTS}

Participant characteristics are summarized according to SOA groups (stratified as $\leq 12 \mathrm{y}$ and $>12 \mathrm{y}$, based on the findings reported below). Earlier SOA was more likely in men and was associated with a higher number of pack-years. In the bivariate analysis, an earlier SOA was also associated with increased risk of CVD events, CVD mortality, and all-cause mortality (Table 1).

\section{Cardiovascular events and smoking-onset age}

During follow-up (median 7.02 years), 210 fatal and non-fatal CVD events were recorded. The non-linear component of the association between SOA and CVD events was statistically significant (p-value=0.002) (Figure 2, panel A). The bootstrapping analyses showed that the best cut-point to define a change in the linear dose-response association was 11-12 years for cardiovascular events (12 years for cardiovascular mortality). Therefore, the analysis was stratified in two SOA groups: $\leq 12 \mathrm{y}$ and $>12 \mathrm{y}$. In the younger group (SOA from 9 to 12 years), each year of delay in SOA was associated with a $29 \%$ decrease in CVD event risk (HR=0.71; 95\% Confidence Interval-CI-: 0.53-0.96) (Table 2). In the older group (SOA from 13 to 30 years), SOA was not associated with CVD risk (HR=1.00; 95\% CI: 0.96-1.04). Similar results were observed in the sensitivity analysis in current and former smokers (Appendix Table A1-A2 and Figure A1-A2) and in men (Appendix Table A3). In women, the 
models in the younger SOA age group did not converge due to the low number of events (Appendix Table A4).

\section{Mortality and smoking onset age}

During follow-up, there were 361 deaths (77 CVD-related). The non-linear component of the association between SOA and CVD mortality was statistically significant ( $p$-value $=0.010$ ) and was non-significant for all-cause mortality (p-value=0.220) (Figure 2, panel B and C).

The bootstrapping analyses showed that the best cut-point to define a change in the linear dose-response association for cardiovascular mortality was 12 years. Therefore, the analysis was stratified in two SOA groups: $\leq 12$ years and $>12$ years. In the younger group, SOA was inversely associated with the risk of CVD mortality. Each year of delay in SOA was associated with a $42 \%$ decrease in CVD mortality risk (HR=0.58; 95\% CI: $0.37-0.90)$ (Table 2). In the older group, SOA was not associated with CVD risk (HR=1.00; 95\% CI: 0.93-1.08). The association between SOA and all-cause mortality was linear. Each year of delay in SOA was associated with $4 \%$ decrease in all-cause mortality risk (HR=0.96; 95\% CI: $0.93-0.98)$ (Table 2). Similar results for both outcomes were observed in the sensitivity analysis in current and former smokers (Appendix Table A1-A2 and Figure A1-A2) and in men (Appendix Table A3).

\section{DISCUSSION}

This study analysed the linear and non-linear dose-response relationship between SOA and three clinical outcomes: CVD events, CVD mortality and all-cause mortality. The association between SOA and CVD fatal and non-fatal events did not follow a linear dose-response association. Two clear but different patterns were observed with a cut-point at 12 years of age. Individuals who started to smoke at or before this cutpoint showed an inverse and linear 
association between SOA and CVD health outcomes. In this group, each year of delay in SOA was associated with a decrease of $29 \%$ and $42 \%$ in CVD events and CVD mortality risk, respectively. In contrast, we did not find a higher SOA-related CVD risk among those who started to smoke after 12 years of age. The association between SOA and all-cause mortality followed a linear pattern: each year of delay in SOA was associated with a $4 \%$ decrease in all-cause mortality. These associations were independent of lifetime cumulative exposure to tobacco.

Several studies have analysed the association between SOA and cardiovascular events and all-cause mortality (19-25). However, in those studies age was categorized prior to the analysis and the linear and non-linear dose-response relationship was not specifically assessed. The use of penalized smoothing splines methods allowed us to explore those patterns of association.

Our results support a clear association between SOA and CVD health outcomes. The magnitude of the association between early SOA and CVD disease risk in our study is consistent with that reported in two large American cohorts (20,24). Choi et al. (20) used data from the United States National Health Interview Survey, in which smoking and CVD clinical events are self-reported. They reported a linear association between SOA $\leq 16$ years and increased risk of CVD-related events: the earlier the SOA, the higher the CVD risk. No association between SOA and CVD risk was observed in the group older than 16 years when they started smoking. In the ARIC Study (24), SOA $\leq 18$ years was related to an increased risk of CVD among current smokers: again, the earlier the SOA, the higher the CVD risk and there was no association in those who started to smoke when older than 18 years. In a European population, Planas et al. also reported a positive association between peripheral arterial disease and SOA $<16$ years, with the study limitations inherent to a retrospective analysis and a small sample size (25). In Asian populations, no clear association between 
early SOA and CVD mortality has been reported; however, the published studies did not evaluate the impact on non-fatal CVD events $(12,26)$. Finally, the Nurses' Health Study did not find increased CVD mortality among women across several SOA groups (23). The differences between these studies could be related to the population of reference: Caucasian $(20,24,25)$ vs Asian $(12,26)$, or only women $(23)$ vs men and women $(20,24,25)$. Moreover, the different SOA cut-points -12 years in our study, 16 years in Choi (20) and Planas et al (25), and 18 years in the ARIC study (24) could be explained by the reliability of selfreported questionnaire data and also by the approach used to define age groups for analysis.

Similarly, the association between early SOA and increased mortality observed in our study is consistent with the findings in previous studies (12,20,23). A reduced mortality risk was associated with older SOA in the follow-up of a cohort in Bangladesh (12) and of participants in the U.S. National Health Interview Surveys (20) and the Nurses' Health Study (23).

Although smoking prevention is important at any age to avoid the additional risks associated with smoking exposure, the results of our study highlight 9 to 12 years as a critical age range. Three main mechanisms have been proposed to explain the relationship between early SOA and health outcomes. First, early initiation might be assumed to lead to higher exposure due to a longer period of exposure (34). In our study, however, the results suggest that an early SOA is an independent risk factor regardless of smoking status at the time of study recruitment or cumulative lifetime exposure to tobacco smoke. Second, as childhood and adolescence are critical periods for organ development, exposure to smoking could affect tissue maturation and ability to adapt to stress, implying an increased risk of future endothelium frailty and propensity to CVD (34). Moreover, early SOA has been associated with higher risk of substance dependence in adulthood (35). 
Smoking remains a key public health issue in Europe (36) and throughout the world (37). Among the factors defining individual exposure to smoking, early SOA has recently emerged as an important variable to consider. In Spain, the average SOA is 14.6 years, affecting $8.9 \%$ of the population aged 14-18 years (38), compared to the European mean SOA of 16.6 years (14). This illustrates the high prevalence of an early SOA in our setting and the need to communicate its consequences and implications in ways that reach young adolescents. Although smoking regulation policies have been shown to be effective in preventing CVD (39), the close relationship between an early SOA and health outcomes recommends the implementation of new strategies focussing on childhood and adolescence.

Our study had several limitations. First, all smoking data were obtained by self-reported questionnaires with no objective assessment of smoking exposure. Nonetheless, a recent study suggests a good correlation between self-reported data and biochemical verification (40). Second, a recall bias may have influenced the quality of information on past smoking exposure (SOA, daily exposure, or quitting date) among former smokers. Third, daily exposure was considered to be constant throughout the lifelong smoking period, which is unlikely to be true for all smokers. Fourth, quitting date was not collected in a consistent format across the three REGICOR surveys. The methods implemented to establish this variable in former smokers may have affected the reported impact of early SOA on the study outcomes. Finally, our study may have been underpowered, especially in women, because of the low number of CVD events and deaths compared to studies in other geographic areas. Furthermore, the population of the Mediterranean region is characterized by a low incidence of CVD events (41).

Our results contribute to the evidence that the age when an individual begins to smoke is an independent risk factor for fatal and non-fatal CVD and for all-cause mortality, independent of cumulative lifelong exposure to smoking. Our results clearly reinforce the 
need to implement health promotion strategies against tobacco use among teenagers and adolescents, especially before they reach 13 years of age. 


\section{ACKNOWLEDGMENTS}

This project was funded by the following sources: Agència de Gestió Ajuts Universitaris de Recerca: 2017 SGR 222, and 2017 SGR 1146; Departament de Salut (PERIS), Generalitat de Catalunya (SLT002/16/00088); ISCIII-FIS-FEDER-ERDF (CIBERCV, PI11 01801, PI08 1327, PI05 1251, PI05 1297, PI02 0471, FIS99/0013-01, FIS96/0026-01, FIS93/0568, FIS92/0009-05); MCIN ISCIII-RETICS-FEDER-ERDF (RD12/0042/0061, RD12/0042/0013, RD06/0009) and Network for Prevention and Health Promotion in primary Care [RedIAPP RD16/0007/0004]. MG was funded by a contract from Instituto de Salud Carlos III FEDER (FIS CP12/03287). IRG was funded by a contract from Pla estratègic de recerca i innovació en salut 2016-2020, Generalitat de Catalunya (SLT006/17/00029).

\section{CONFLICT OF INTEREST}

The authors declare they do not have any conflict of interest to disclose. 


\section{REFERENCES}

1. Gordon P, Flanagan P. Smoking: A risk factor for vascular disease. J Vasc Nurs. 2016;34:79-86.

2. Ockene IS, Miller NH. Cigarette Smoking, Cardiovascular Disease, and Stroke. Circulation. 1997;96:3243-7.

3. McEvoy JW, Nasir K, DeFilippis AP, et al. Relationship of Cigarette Smoking With Inflammation and Subclinical Vascular DiseaseSignificance. Arterioscler Thromb Vasc Biol. 2015;35:1002-10.

4. Stallones RA. The association between tobacco smoking and coronary heart disease. Int J Epidemiol. 2015;44:735-43.

5. Kelly TN, Gu D, Chen J, et al. Cigarette smoking and risk of stroke in the chinese adult population. Stroke. 2008;39:1688-93.

6. Criqui MH, Aboyans V. Epidemiology of Peripheral Artery Disease. Circ Res. 2015;116:1509-26.

7. Tang W, Yao L, Roetker NS, et al. Lifetime Risk and Risk Factors for Abdominal Aortic Aneurysm in a 24-Year Prospective Study: The ARIC Study (Atherosclerosis Risk in Communities). Arterioscler Thromb Vasc Biol. 2016;36:2468-77.

8. Shiraishi N, Kitamura K, Kohda Y, et al. Prevalence and risk factor analysis of nephrosclerosis and ischemic nephropathy in the Japanese general population. Clin Exp Nephrol. 2014;18:461-8.

9. Hohenwalter EJ. Chronic mesenteric ischemia: diagnosis and treatment. Semin Intervent Radiol. 2009;26:345-51.

10. Landenhed M, Engström G, Gottsäter A, et al. Risk profiles for aortic dissection and ruptured or surgically treated aneurysms: a prospective cohort study. J Am Heart Assoc. 2015;4:e001513. 
11. Doll R, Peto R, Boreham J, Sutherland I. Mortality in relation to smoking: 50 years' observations on male British doctors. BMJ. 2004;328:1519-0.

12. Wu F, Chen Y, Parvez F, et al. A Prospective Study of Tobacco Smoking and Mortality in Bangladesh. PLoS One. 2013;8:e58516.

13. Mons U, Müezzinler A, Gellert C, et al. Impact of smoking and smoking cessation on cardiovascular events and mortality among older adults: meta-analysis of individual participant data from prospective cohort studies of the CHANCES consortium. BMJ. 2015;350:h1551.

14. Dani JA, Harris RA. Nicotine addiction and comorbidity with alcohol abuse and mental illness. Nat Neurosci. 2005;8:1465-70.

15. Genuneit J, Weinmayr G, Radon K, et al. Smoking and the incidence of asthma during adolescence: results of a large cohort study in Germany. Thorax. 2006;61:572-8.

16. Wiencke JK, Kelsey KT. Teen smoking, field cancerization, and a "critical period" hypothesis for lung cancer susceptibility. Environ Health Perspect. 2002;110:555-8.

17. Peto R, Darby S, Deo H, et al. Smoking, smoking cessation, and lung cancer in the UK since 1950: combination of national statistics with two case-control studies. BMJ. 2000;321:323-9.

18. Funatogawa I, Funatogawa T, Yano E. Impacts of early smoking initiation: long-term trends of lung cancer mortality and smoking initiation from repeated cross-sectional surveys in Great Britain. BMJ Open. 2012;2:e001676.

19. Choi SH, Stommel M. Impact of Age at Smoking Initiation on Smoking-Related Morbidity and All-Cause Mortality. Am J Prev Med. 2017:53:33-41.

20. Catsburg C, Miller AB, Rohan TE. Active cigarette smoking and risk of breast cancer. Int J Cancer. 2015;136:2204-9.

21. Park S, Jee SH, Shin H-R, et al. Attributable fraction of tobacco smoking on cancer 
using population-based nationwide cancer incidence and mortality data in Korea. BMC Cancer. 2014;14:406.

22. Kenfield SA, Stampfer MJ, Rosner BA, Colditz GA. Smoking and Smoking Cessation in Relation to Mortality in Women. JAMA. 2008;299:2037.

23. Huxley RR, Yatsuya H, Lutsey PL, et al. Impact of Age at Smoking Initiation, Dosage, and Time Since Quitting on Cardiovascular Disease in African Americans and Whites: The Atherosclerosis Risk in Communities Study. Am J Epidemiol. 2012;175:816-26.

24. Planas A, Clará A, Marrugat J, et al. Age at onset of smoking is an independent risk factor in peripheral artery disease development. J Vasc Surg Off Publ Soc Vasc Surg [and] Int Soc Cardiovasc Surgery, North Am Chapter. 2002;35:506-9.

25. Honjo K, Iso $\mathrm{H}$, Tsugane $\mathrm{S}$, et al. The effects of smoking and smoking cessation on mortality from cardiovascular disease among Japanese: pooled analysis of three largescale cohort studies in Japan. Tob Control. 2010;19:50-7.

26. Filippidis FT, Agaku IT, Vardavas CI. The association between peer, parental influence and tobacco product features and earlier age of onset of regular smoking among adults in 27 European countries. Eur J Public Health. 2015;25:814-8.

27. Grau M, Subirana I, Elosua R, et al. Trends in cardiovascular risk factor prevalence (1995-2000-2005) in northeastern Spain. Eur J Cardiovasc Prev Rehabil. 2007;14:6539.

28. Manual of the MONICA Project. Geneva: World Health Organitzation. Available at: http://www.thl.fi/publications/monica/index.html. Accessed June 20, 2017

29. Baena-Díez JM, Alzamora-Sas MT, Grau M, et al. Validity of the MONICA cardiovascular questionnaire compared with clinical records. Gac Sanit. 2009;23:519_ 25.

30. Kozlowski LT, Dollar KM, Giovino GA. Cigar/Cigarillo Surveillance. Am J Prev Med. 
$2008 ; 34: 424-6$.

31. R Core Team (2018). R: A language and environment for statistical computing. R Foundation for Statistical Computing, Vienna, Austria. URL https://www.Rproject.org/.

32. Velescu A, Clara A, Martí R, et al. Abnormally High Ankle-Brachial Index is Associated with All-cause and Cardiovascular Mortality: The REGICOR Study. Eur J Vasc Endovasc Surg. 2017;54:370-7.

33. Eilers PH, Marx BD. Flexible smoothing with B-splines and penalties. Statistical Science 1996;11:89-121.

34. Gall S, Huynh QL, Magnussen CG, et al. Exposure to parental smoking in childhood or adolescence is associated with increased carotid intima-media thickness in young adults: evidence from the Cardiovascular Risk in Young Finns study and the Childhood Determinants of Adult Health Study. Eur Heart J. 2014;35:2484-91.

35. Dani JA, Harris RA. Nicotine addiction and comorbidity with alcohol abuse and mental illness. Nat Neurosci. 2005;8:1465-70.

36. Wilkins E, Wilson L, Wickramasinghe K, et al. European Cardiovascular Disease Statistics 2017. European Heart Network, Brussels

37. GBD 2016 Risk Factors Collaborators. Global Health Metrics Global, regional, and national comparative risk assessment of 84 behavioural, environmental and occupational, and metabolic risks or clusters of risks, 1990-2016: a systematic analysis for the Global Burden of Disease Study 2016. Lancet. 2017;390:1345-422.

38. Observatorio Español de la Droga y las Toxicomanías. Informe 2016. Alcohol, tabaco y drogas ilegales en España. Plan Nacional sobre Drogras, Madrid.

39. Agüero F, Dégano IR, Subirana I, et al. Impact of a partial smoke-free legislation on myocardial infarction incidence, mortality and case-fatality in a population-based 
registry: the REGICOR Study. PLoS ONE 2013;8:e53722.

40. Caraballo RS, Giovino GA, Pechacek TF, Mowery PD. Factors associated with discrepancies between self-reports on cigarette smoking and measured serum cotinine levels among persons aged 17 years or older: Third National Health and Nutrition Examination Survey, 1988-1994. Am J Epidemiol. 2001;153:807-14.

41. Liyanage $\mathrm{T}$, Ninomiya $\mathrm{T}$, Wang $\mathrm{A}$, et al. Effects of the Mediterranean Diet on Cardiovascular Outcomes-A Systematic Review and Meta-Analysis. PLoS One. 2016;11:e0159252. 


\section{FIGURES}

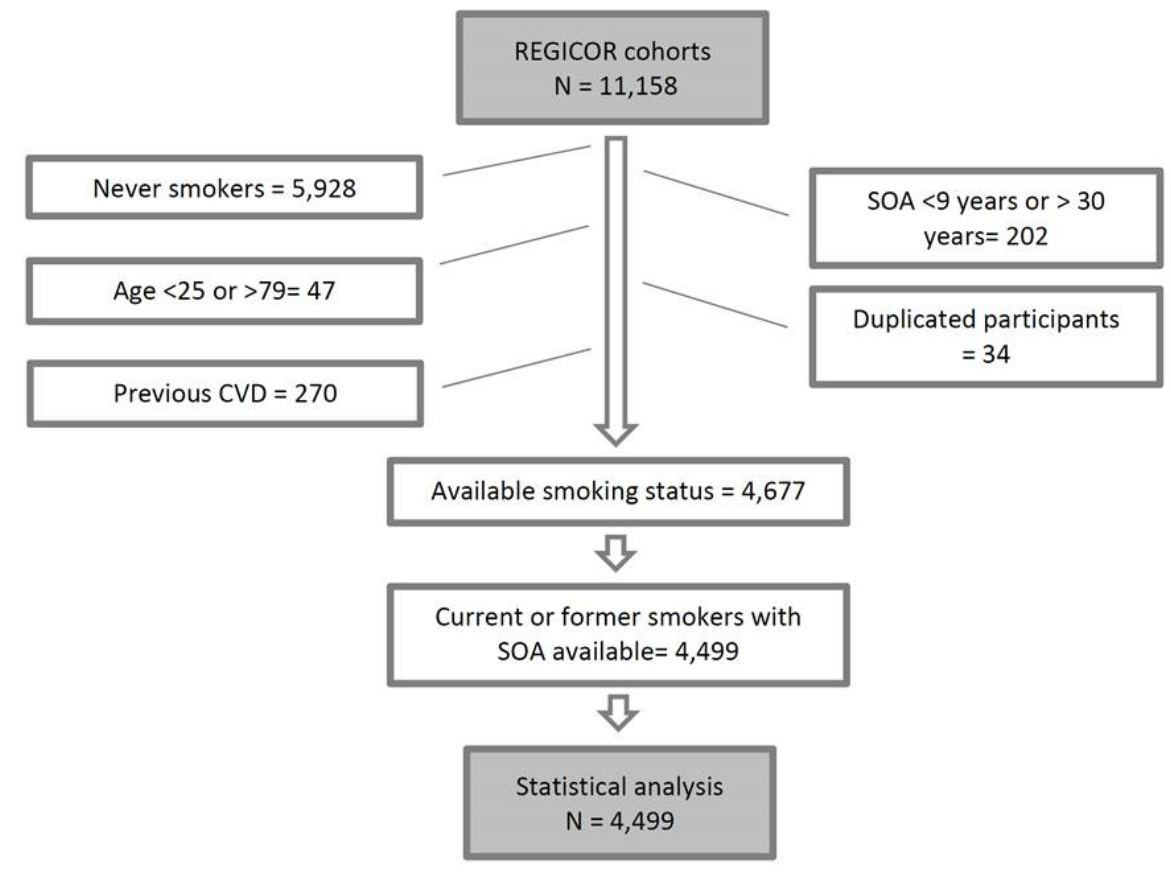

Figure 1. Flow chart of participant selection and inclusion. REGICOR population-based cohort study, participants recruited from 1995 to 2005, aged 25 to 79 years, with a median 7.02 years of follow-up. 

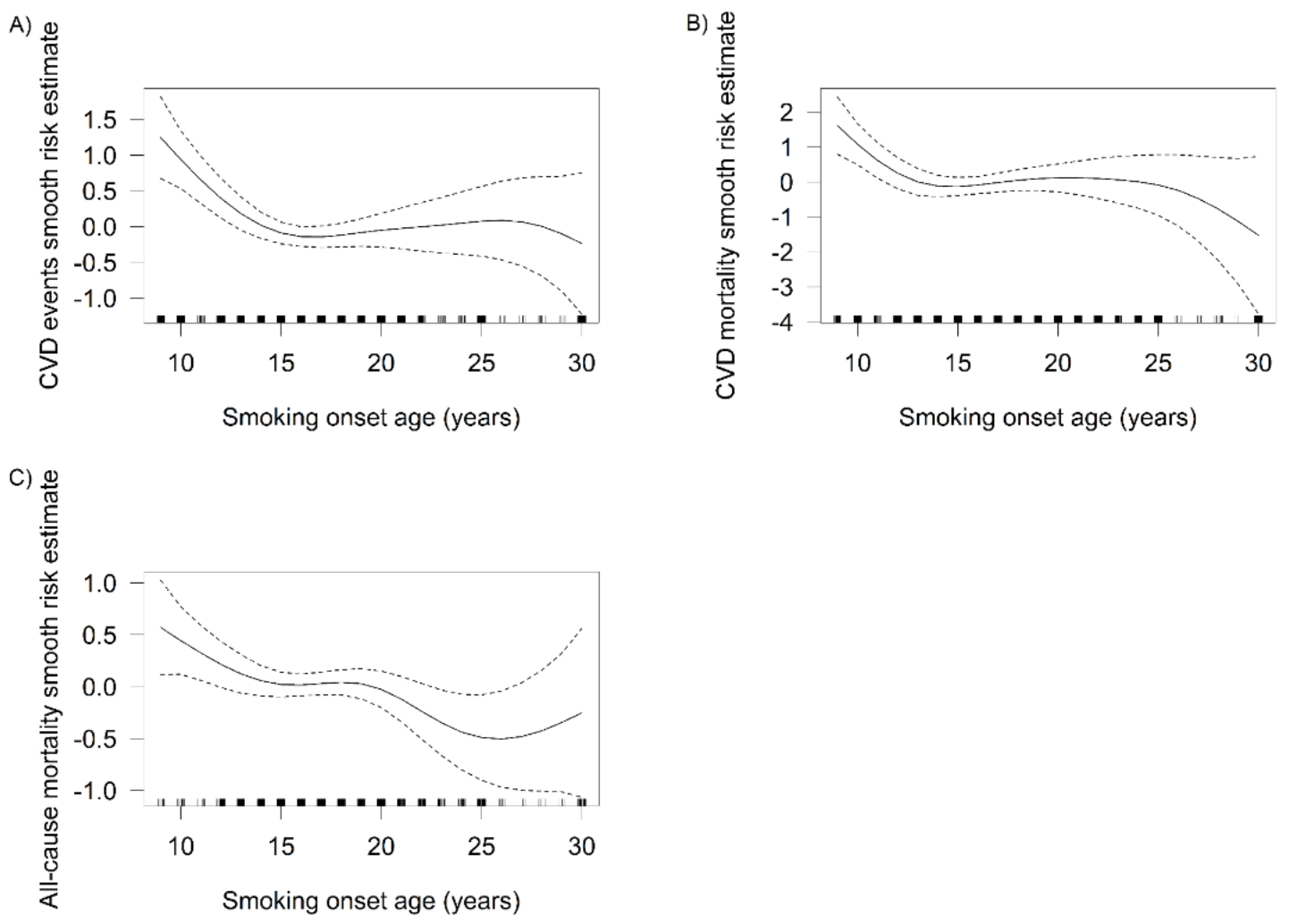

Figure 2. Penalized smoothing spline plots of the linear and non-linear dose-response association between smoking onset age and the outcomes of interest: A.-Cardiovascular events; B.-Cardiovascular mortality; C.-All-cause mortality. REGICOR population-based cohort study, participants recruited from 1995 to 2005, aged 25 to 79 years, with a median 7.02 years of follow-up.

Figure 2 footnote: The lines along the horizontal axes represent the number of individuals across smoking onset age. 
Table 1. Baseline characteristics of the study population according to smoking onset age.

REGICOR population-based cohort study, participants recruited from 1995 to 2005, aged 25 to 79 years, with a median 7.02 years of follow-up.

\begin{tabular}{|c|c|c|c|}
\hline Variables & (n) & $\begin{array}{c}\text { Smoking onset } \\
\text { age } \leq \mathbf{1 2 y} \\
(\mathrm{n}=244) \\
\end{array}$ & $\begin{array}{c}\text { Smoking onset } \\
\text { age }>\mathbf{1 2 y} \\
(\mathrm{n}=4,255) \\
\end{array}$ \\
\hline Age, years * & 4499 & $57.7(12.3)$ & $49.9(12.7)$ \\
\hline Sex: women, n (\%) & 4499 & $19(7.8 \%)$ & $1389(32.6 \%)$ \\
\hline Education, n (\%) & 4447 & - & - \\
\hline University & & $13(5.5 \%)$ & $895(21.3 \%)$ \\
\hline Secondary school & & $43(18.2 \%)$ & $1263(30.0 \%)$ \\
\hline Primary school & & $180(73.3 \%)$ & $2053(48.8 \%)$ \\
\hline Body mass index $*, \mathrm{Kg} / \mathrm{m}^{2}$ & 4466 & $27.3(4.7)$ & $26.8(4.3)$ \\
\hline Diabetes, n (\%) & 4427 & $47(19.5 \%)$ & $429(10.2 \%)$ \\
\hline Glucose serum*, $m g / d L$ & 4390 & $108(28.9)$ & $101(26.0)$ \\
\hline Hypertension, $\mathrm{n}(\%)$ & 4488 & $135(56.5 \%)$ & $1564(37.5 \%)$ \\
\hline$S B P^{*}, m m H g$ & 4485 & $136(19.5)$ & $126(19.7)$ \\
\hline$D B P^{*}, m m H g$ & 4474 & $80.4(10.7)$ & $78.3(10.7)$ \\
\hline Total cholesterol* ${ }^{*}, \mathrm{mg} / \mathrm{dL}$ & 4386 & $213(43.5)$ & $213(42.0)$ \\
\hline HDL cholesterol* ${ }^{*}, \mathrm{mg} / \mathrm{dL}$ & 4373 & $49.4(13.1)$ & $49.4(13.1)$ \\
\hline LDL cholesterol*, mg/dL & 4214 & $139(39.4)$ & $140(37.6)$ \\
\hline Triglycerides $\dagger, \mathrm{mg} / \mathrm{dL}$ & 4379 & $103[77 ; 143]$ & $97[72 ; 136]$ \\
\hline \multicolumn{4}{|l|}{ Smoking exposure } \\
\hline Smoking status n (\%) & 4499 & - & - \\
\hline Current smoker & & $111(45.5 \%)$ & $2224(52.3 \%)$ \\
\hline Former smoker $>1 y$ & & $133(54.5 \%)$ & $2031(47.7 \%)$ \\
\hline Pack-years $\dagger$ & 3100 & $32.7[9.2 ; 55.6]$ & $14.0[3.6 ; 28.5]$ \\
\hline Years since quitting* & 3119 & $5.4(8.9)$ & $2.9(6.7)$ \\
\hline \multicolumn{4}{|l|}{ Outcomes } \\
\hline Fatal/non-fatal CVD event, n (\%) & 4499 & $34(13.9 \%)$ & $176(4.1 \%)$ \\
\hline CVD mortality, n (\%) & 4499 & $17(7.0 \%)$ & $60(1.4 \%)$ \\
\hline All-cause mortality, n (\%) & 4499 & $59(24.2 \%)$ & $302(7.1 \%)$ \\
\hline
\end{tabular}

$*$ mean (standard deviation); $\dagger$ median [interquartile range]

SBP: systolic blood pressure; DBP: diastolic blood pressure; HDL: high density lipoprotein;

LDL: low density lipoprotein; CVD: cardiovascular disease 
Table 2. Multivariate adjusted association between smoking onset age, as a continuous variable, and the outcomes of interest in current and former smokers. REGICOR populationbased cohort study, participants recruited from 1995 to 2005 , aged 25 to 79 years, with a median 7.02 years of follow-up.

\begin{tabular}{|c|c|c|}
\hline & \multicolumn{2}{|c|}{ Smoking onset age } \\
\hline & $\begin{array}{c}\text { Smoking onset age } \\
\text { between } 9 \text { to } 12 \text { years } \\
(n=219)\end{array}$ & $\begin{array}{c}\text { Smoking onset age } \\
\text { between } 13 \text { to } 30 \text { years } \\
(n=3952)\end{array}$ \\
\hline \multicolumn{3}{|l|}{$\begin{array}{c}\text { CVD events: fatal and } \\
\text { non-fatal }\end{array}$} \\
\hline HR & $0.71^{\mathrm{a}}$ & $1.00^{b}$ \\
\hline $95 \% \mathrm{CI}$ & $0.53-0.96$ & $0.96-1.04$ \\
\hline \multicolumn{3}{|l|}{ CVD mortality } \\
\hline HR & $0.58^{c}$ & $1.00^{\mathrm{d}}$ \\
\hline \multirow[t]{2}{*}{$95 \% \mathrm{CI}$} & $0.37-0.90$ & $0.93-1.08$ \\
\hline & \multicolumn{2}{|c|}{$\begin{array}{l}\text { Smoking onset age between } 9 \text { to } 30 \text { years } \\
(\mathrm{n}=4171)\end{array}$} \\
\hline \multicolumn{3}{|l|}{ All-cause mortality } \\
\hline HR & \multicolumn{2}{|c|}{$0.96^{\mathrm{e}}$} \\
\hline $95 \% \mathrm{CI}$ & \multicolumn{2}{|c|}{$0.93-0.98$} \\
\hline
\end{tabular}

CVD: cardiovascular disease; HR: hazard ratio; CI: confidence interval

a. Adjusted for age, total cholesterol, high-density lipoprotein cholesterol, smoking status, pack-years, recruitment survey and years since quitting smoking.

b. Adjusted for age, glucose, high-density lipoprotein cholesterol, smoking status, pack-years, recruitment survey and years since quitting smoking.

c. Adjusted for age, hypertension treatment, smoking status, pack-years, recruitment survey and years since quitting smoking.

d. Adjusted for age, glucose, smoking status, pack-years, recruitment survey and years since quitting smoking

e. Adjusted for age, sex, glucose, smoking status, pack-years, recruitment survey and years since quitting smoking 


\section{APPENDIX A}

Appendix Table A1. Multivariate adjusted association between smoking onset age, as a continuous variable, and the outcomes of interest in current smokers. REGICOR populationbased cohort study, participants recruited from 1995 to 2005, aged 25 to 79 years, with a median 7.02 years of follow-up.

\begin{tabular}{|c|c|c|}
\hline & \multicolumn{2}{|c|}{ Smoking onset age in current smokers } \\
\hline & $\begin{array}{c}\text { Smoking onset age } \\
\text { between } 9 \text { and } 12 \text { years } \\
\qquad(n=103)\end{array}$ & $\begin{array}{c}\text { Smoking onset age } \\
\text { between } 13 \text { and } 30 \text { years } \\
(n=2072)\end{array}$ \\
\hline \multicolumn{3}{|l|}{ CVD events: fatal and non-fatal } \\
\hline $\mathrm{HR}$ & $0.57^{\mathrm{a}}$ & $0.99^{b}$ \\
\hline $95 \% \mathrm{Cl}$ & $0.34-0.94$ & $0.92-1.06$ \\
\hline \multicolumn{3}{|l|}{ CVD mortality } \\
\hline HR & $0.47^{c}$ & $1.04^{d}$ \\
\hline $95 \% \mathrm{Cl}$ & $0.14-1.60$ & $0.93-1.17$ \\
\hline & \multicolumn{2}{|c|}{$\begin{array}{l}\text { Smoking onset age between } 9 \text { and } \mathbf{3 0} \text { years } \\
\qquad(\mathrm{n}=2175)\end{array}$} \\
\hline \multicolumn{3}{|l|}{ All-cause mortality } \\
\hline $\mathrm{HR}$ & \multicolumn{2}{|c|}{$0.95^{\mathrm{e}}$} \\
\hline $95 \% \mathrm{Cl}$ & \multicolumn{2}{|c|}{$0.91-1.00$} \\
\hline
\end{tabular}

CVD: cardiovascular disease; HR: hazard ratio; Cl: confidence interval

a. Adjusted for age, triglycerides, pack-years and recruitment survey.

b. Adjusted for age, hypertension treatment, glucose, total cholesterol, high-density lipoprotein, body mass index, pack-years and recruitment survey.

c. Adjusted for age, diastolic blood pressure, pack-years and recruitment survey.

d. Adjusted for age, hypertension treatment, diabetes treatment, pack-years and recruitment survey.

e. Adjusted for age, sex, glucose, pack-years and recruitment survey. 
Appendix Table A2. Multivariate adjusted association between smoking onset age, as a continuous variable, and the outcomes of interest in former smokers. REGICOR populationbased cohort study, participants recruited from 1995 to 2005, aged 25 to 79 years, with a median 7.02 years of follow-up.

\begin{tabular}{|c|c|c|}
\hline & \multicolumn{2}{|c|}{ Smoking onset age in former smokers } \\
\hline & $\begin{array}{c}\text { Smoking onset age } \\
\text { between } 9 \text { and } 12 \text { years } \\
(n=116)\end{array}$ & $\begin{array}{c}\text { Smoking onset age } \\
\text { between } 13 \text { and } 30 \text { years } \\
(n=1880)\end{array}$ \\
\hline \multicolumn{3}{|l|}{ CVD events: fatal and non-fatal } \\
\hline HR & $0.62^{\mathrm{a}}$ & $1.01^{b}$ \\
\hline $95 \% \mathrm{Cl}$ & $0.41-0.94$ & $0.95-1.07$ \\
\hline \multicolumn{3}{|l|}{ CVD mortality } \\
\hline HR & $0.46^{c}$ & $0.98^{d}$ \\
\hline $95 \% \mathrm{Cl}$ & $0.27-0.78$ & $0.89-1.08$ \\
\hline & \multicolumn{2}{|c|}{$\begin{array}{l}\text { Smoking onset age between } 9 \text { and } 30 \text { years } \\
\qquad(n=1996)\end{array}$} \\
\hline \multicolumn{3}{|l|}{ All-cause mortality } \\
\hline $\mathrm{HR}$ & \multicolumn{2}{|c|}{$0.96^{\mathrm{e}}$} \\
\hline $95 \% \mathrm{Cl}$ & \multicolumn{2}{|c|}{$0.92-1.00$} \\
\hline
\end{tabular}

CVD: cardiovascular disease; HR: hazard ratio; Cl: confidence interval

a. Adjusted for age, hypertension treatment, diabetes treatment, pack-years, years since quitting and recruitment survey.

b. Adjusted for age, diabetes, pack-years, years since quitting and recruitment survey.

c. Adjusted for age, systolic blood pressure, hypertension treatment, pack-years, years since quitting and recruitment survey.

d. Adjusted for age, diabetes, pack-years, years since quitting and recruitment survey.

e. Adjusted for age, glucose, pack-years, years since quitting and recruitment survey. 
Appendix Table A3. Multivariate adjusted association between smoking onset age, as a continuous variable, and the outcomes of interest in men. REGICOR population-based cohort study, participants recruited from 1995 to 2005, aged 25 to 79 years, with a median 7.02 years of follow-up.

\begin{tabular}{|c|c|c|}
\hline & \multicolumn{2}{|c|}{$\begin{array}{l}\text { Smoking onset age in former and current smokers. } \\
\text { Men only }\end{array}$} \\
\hline & $\begin{array}{c}\text { Smoking onset age } \\
\text { between } 9 \text { and } 12 \text { years } \\
(n=202)\end{array}$ & $\begin{array}{c}\text { Smoking onset age } \\
\text { between } 13 \text { and } \mathbf{3 0} \text { years } \\
(n=2654)\end{array}$ \\
\hline \multicolumn{3}{|l|}{ CVD events: fatal and non-fatal } \\
\hline $\mathrm{HR}$ & $0.73^{a}$ & $1.00^{b}$ \\
\hline $95 \% \mathrm{Cl}$ & $0.54-0.98$ & $0.96-1.04$ \\
\hline \multicolumn{3}{|l|}{ CVD mortality } \\
\hline$H R$ & $0.58^{c}$ & $1.02^{d}$ \\
\hline $95 \% \mathrm{Cl}$ & $0.37-0.91$ & $0.95-1.10$ \\
\hline & \multicolumn{2}{|c|}{$\begin{array}{l}\text { Smoking onset age between } 9 \text { and } 30 \text { years } \\
\qquad(n=2856)\end{array}$} \\
\hline \multicolumn{3}{|l|}{ All-cause mortality } \\
\hline $\mathrm{HR}$ & \multicolumn{2}{|c|}{$0.95^{\mathrm{e}}$} \\
\hline $95 \% \mathrm{Cl}$ & \multicolumn{2}{|c|}{$0.92-0.98$} \\
\hline
\end{tabular}

CVD: cardiovascular disease; HR: hazard ratio; Cl: confidence interval

a. Adjusted for age, hypertension treatment, diabetes treatment, pack-years, years since quitting and recruitment survey.

b. Adjusted for age, diabetes, pack-years, years since quitting and recruitment survey.

c. Adjusted for age, systolic blood pressure, hypertension treatment, pack-years, years since quitting and recruitment survey.

d. Adjusted for age, diabetes, pack-years, years since quitting and recruitment survey.

e. Adjusted for age, glucose, pack-years, years since quitting and recruitment survey. 
Appendix Table A4. Multivariate adjusted association between smoking onset age, as a continuous variable, and the outcomes of interest in women. REGICOR population-based cohort study, participants recruited from 1995 to 2005, aged 25 to 79 years, with a median 7.02 years of follow-up.

\begin{tabular}{|c|c|c|}
\hline & \multicolumn{2}{|c|}{$\begin{array}{l}\text { Smoking onset age in former and current smokers. } \\
\text { Women only }\end{array}$} \\
\hline & $\begin{array}{c}\text { Smoking onset age } \\
\text { between } 9 \text { and } 12 \text { years } \\
(n=17)\end{array}$ & $\begin{array}{c}\text { Smoking onset age } \\
\text { between } \mathbf{1 3} \text { and } \mathbf{3 0} \text { years } \\
(n=1298)\end{array}$ \\
\hline \multicolumn{3}{|l|}{ CVD events: fatal and non-fatal } \\
\hline $\mathrm{HR}$ & --- & $0.98^{\mathrm{a}}$ \\
\hline $95 \% \mathrm{Cl}$ & --- & $0.84-1.15$ \\
\hline \multicolumn{3}{|l|}{ CVD mortality } \\
\hline$H R$ & --- & --- \\
\hline $95 \% \mathrm{Cl}$ & --- & --- \\
\hline & \multicolumn{2}{|c|}{$\begin{array}{l}\text { Smoking onset age between } 9 \text { and } \mathbf{3 0} \text { years } \\
\qquad(n=1315)\end{array}$} \\
\hline \multicolumn{3}{|l|}{ All-cause mortality } \\
\hline $\mathrm{HR}$ & \multicolumn{2}{|c|}{$1.03^{b}$} \\
\hline $95 \% \mathrm{Cl}$ & \multicolumn{2}{|c|}{$0.91-1.17$} \\
\hline
\end{tabular}

CVD: cardiovascular disease; HR: hazard ratio; Cl: confidence interval

a. Adjusted for age, diabetes, pack-years, years since quitting and recruitment survey.

b. Adjusted for age, glucose, pack-years, years since quitting and recruitment survey. 

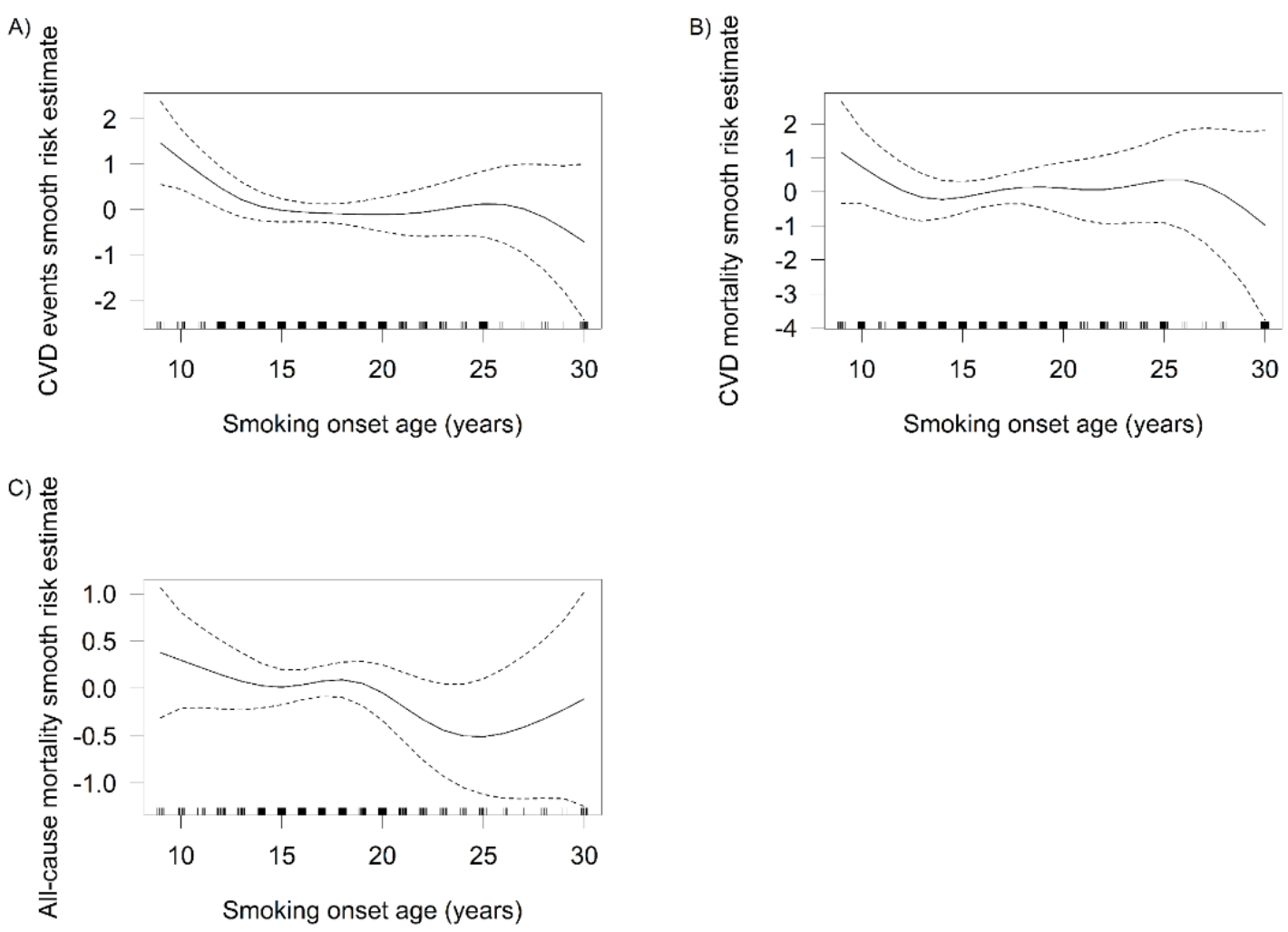

Appendix Figure A1. Current smokers: Smoothing spline plots of the linear and non-linear dose-response association between smoking onset age and the outcomes of interest (A.Cardiovascular events; B.-Cardiovascular mortality; C.-All-cause mortality). REGICOR population-based cohort study, participants recruited from 1995 to 2005, aged 25 to 79 years, with a median 7.02 years of follow-up.

Appendix Figure Al footnote: The lines along the horizontal axes represent the number of current smokers according to smoking onset age. 

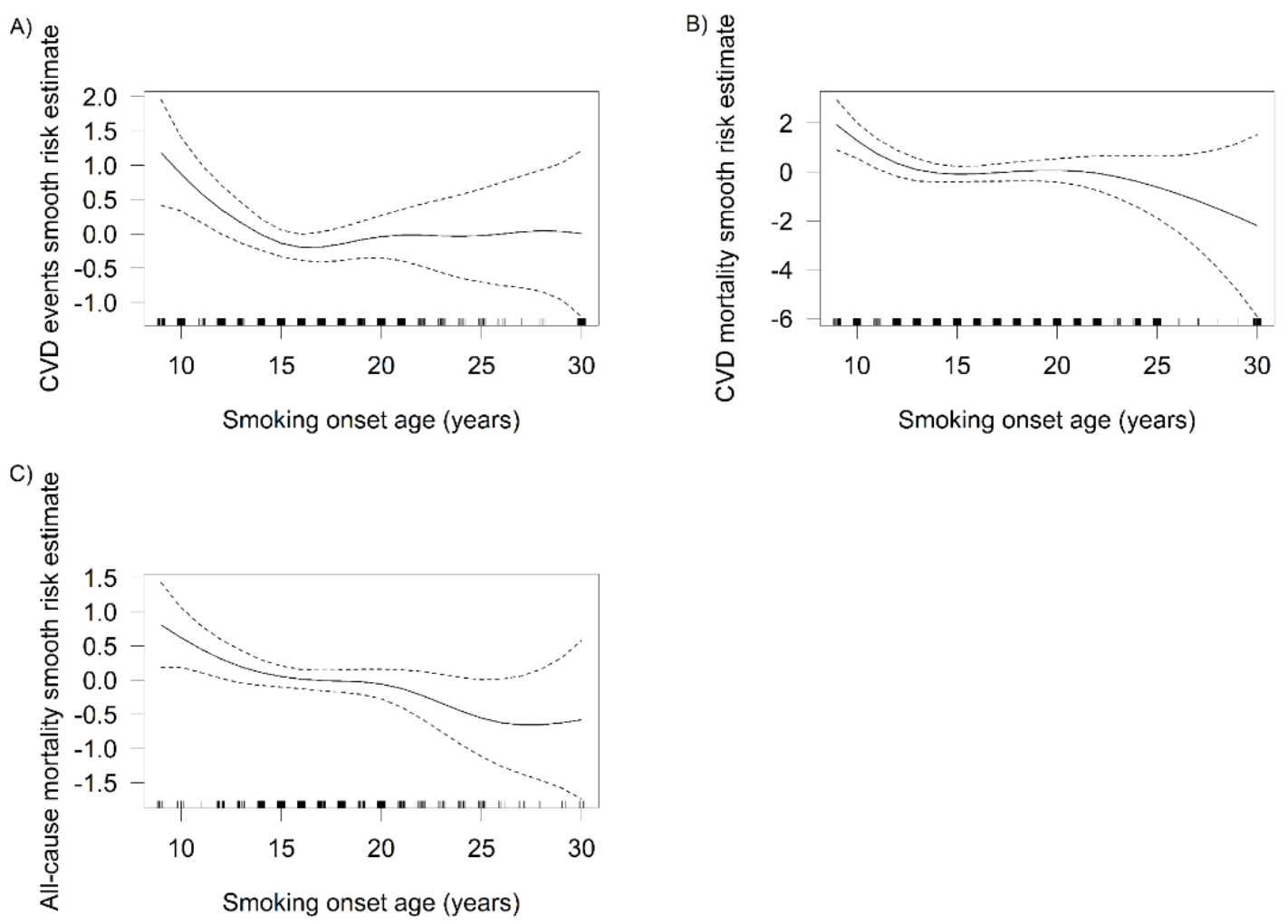

Appendix Figure A2. Former smokers: Smoothing spline plots of the linear and non-linear dose-response association between smoking onset age and the outcomes of interest (A.Cardiovascular events; B.-Cardiovascular mortality; C.-All-cause mortality). REGICOR population-based cohort study, participants recruited from 1995 to 2005, aged 25 to 79 years, with a median 7.02 years of follow-up.

Appendix Figure A2 footnote: The lines along the horizontal axes represent the number of former smokers according to smoking onset age. 